\title{
The effects of placement on dementia care recipients' and family caregivers' quality of life: a literature review
}

\author{
Heehyul Moon, Peggye Dilworth-Anderson and Johannes Gräske
}

\author{
Heehyul Moon is an Assistant \\ Professor at the Raymond \\ A. Kent School of Social Work, \\ University of Louisville, \\ Louisville, Kentucky, USA. \\ Peggye Dilworth-Anderson is a \\ Professor at the Department of \\ Health Policy and \\ Management, Gillings School \\ of Global Public Health, \\ University of North Carolina at \\ Chapel Hill, Chapel Hill, \\ North Carolina, USA. \\ Johannes Gräske is a \\ Professor for Clinical Nursing \\ Research and Evaluation at the \\ Alice Salomon Hochschule \\ Berlin, University of Applied \\ Sciences, Berlin, Germany.
}

\begin{abstract}
Purpose - The purpose of this paper is to review and synthesize the research literature on the quality of life (QoL) of both caregivers (CGs) and care recipients (CRs) with dementia after admission to long-term care facilities.

Design/methodology/approach - Four databases - AgeLine, Medline, EBSCO, and PyscINFO - were searched and the relevant literature from 2002 onwards was reviewed.

Findings - The review of 12 studies (five studies, including only family CGs; six studies including residents; one study including both family CGs and CRs) reveals a discrepancy regarding the effects of institutionalization on the CRs' and CGs' QoL. Among seven studies on CRs' QoL change, some reviewed studies found a significant decline in CRs' QoL after placement with others showing that CRs' QoL was improved or stable. While some reports indicated that some family CGs benefited from placement, others showed that CGs merely maintained their QoL. However, family CGs in the reviewed studies were more likely to report improved QoL than were their CRs after institutionalization.

Research limitations/implications - The authors recommend that future studies should focus on understanding the individual's adaptation to placement, dyadic changes in QoL (including mediators/moderators). They emphasize the need for a comprehensive longitudinal study with more than one wave and includes diverse groups including racial/ethnic minority CGs and CRs.

Originality/value - This study reviewed and synthesized the research literature on the QoL of both caregiver and the people with dementia they cared for after those they cared for were admitted to long-term care facilities. The conclusions drawn about influences on QoL provide guidance for identifying best practices and research.
\end{abstract}

Keywords Literature review, Caregiving, Quality of life, Dementia, Family, Institution

Paper type Literature review

\section{Introduction}

The world's aging population (age $>60$ years) is dramatically increasing (United Nations, 2013). The oldest-old group (age $>75-85$ years) is currently the fastest growing population in many countries, including China, the USA, India, Japan, Germany, and Russia, and it is expected to increase 151 percent between 2005 and 2030 (Dobriansky et al., 2007). Since advancing age remains the greatest risk factor for dementia, especially Alzheimer's disease (AD), such a population trend implies that there will be an increase in need for care by the elderly with AD. As of 2013, there were an estimated 44.4 million people with dementia worldwide. This number will increase to an estimated 75.6 million in 2030 and to 135.5 million in 2050 (Prince et al., 2013).

In the USA, over 34 million people are elderly and 20 percent of the population will be elderly by 2030. More than five million people age 65 and older suffer from AD, and this group accounts for approximately 60 to 80 percent of all dementia cases. The number of individuals in the USA suffering from $A D$ is expected to reach between 7.7 and 9 million by 2030 as the baby boomers age (Alzheimer's Association, 2014). Individuals with dementia from the baby boomer and subsequent generations in the USA will face a shortage of family CGs as the CG-to-care receiver
Received 22 January 2016 Revised 11 April 2016

15 June 2016

13 July 2016

Accepted 2 August 2016 
support ratio shifts from 7 for every person in high-risk years of 80 -plus to 4 to 1 by 2030 and then to less than 3 to 1 by 2050 (Redfoot et al., 2013). These projections emphasize the great need for residential care for the elderly with dementia.

Over three-fourths of older adults with dementia live in long-term care facilities, such as nursing homes, residential-care/assisted-living facilities (RC/AL), or group homes (Macdonald et al., 2002; Prince et al., 2013). Skilled nursing facilities (SNFs), based on a medical model, traditionally have served the majority of older adults with $\mathrm{AD}$ and other related dementias. Recently, however, more family-like environments, such as RC/ALs, are gaining attention in the USA (Sloane et al., 2005). The driving concept of an RC/AL is the enhancement of residents' independence, autonomy, dignity, and privacy by improving their quality of life (QoL) as well as that of their family and by improving staff satisfaction (Sloane et al., 2005). Interest in group homes for people with dementia has been growing in other countries, including Sweden, the Netherlands, Japan, and Germany (Boekhorst et al., 2008; Funaki et al., 2005). Such facilities provide home-like environments with small numbers of residents and staff. Studies have noted that group homes respect individual values and lifestyles and focus on rehabilitating residents' daily living skills to improve independence and self-determination (Funaki et al., 2005; Suzuki et al., 2008).

With increasing numbers of dementia patients living in long-term care settings, the re is a growing need to examine the QoL of patients and family CGs after placement. The concept of QoL is defined broadly and measured in various ways (Logsdon et al., 2002). According to the World Health Organization, QoL is "an individual's perceptions of their position in life in the context of the culture and values systems in which they live, and in relationship to their goals, expectations, and standards and concerns" (Whoqol Group, 1995, p. 1). Thus, QoL, to some researchers, reflects characteristics of both individuals and their environment (adjustment and culture) (e.g. Ferrans et al., 2005; Kitrungrote and Cohen, 2006). Thus Logsdon et al. (2002) conceptualize QoL as comprised of behavioral competence, psychological status, physical functioning, and interpersonal environment.

More specific to the research reported in this paper, Ready et al. (2004) suggest that despite there being no consensus definition of QoL of people with dementia, mood and affect are agreed to be important components. The CR's ability to "experience positive emotion, feeling of belonging, and enjoyment" and care recipients' (CRs) "relative absence of experiencing negative emotion" are critical components of QOL in dementia (p. 257). Both broad and narrow QOL constructs have also been introduced in the context of people with Alzheimer's disease and related dementia, with the dimensions used in these models being similar to Lawton's (1994) definition and include both subjective and objective domains. For example, according to Brod et al. (1999), as a narrower construct excluding other physical symptoms of disease, QoL in people with Alzheimer's disease includes context (Lawton's environmental and behavioral competence), functioning and behavior (Lawton's behavioral competence), and dementia-specific QoL (Lawton's psychological well-being).

\section{Long-term care placement and quality of life}

Relief from caregiving is a general expectation when a family member is placed in long-term care. However, studies suggest that family CGs of people with dementia remain involved both physically and emotionally in giving care albeit in new and different ways after placement: visiting, interacting with staff as well as their relatives, and making decisions about finances and health care (Gaugler et al., 2010; Zarit and Whitlatch, 1992). Thus, their QoL may continue to be affected. The extended stress process model of family caregiving of demented relatives in institutional settings (Whitlatch et al., 2001) explains how these family CGs experience stress after admission. The authors suggest that these family CGs may be influenced continuously after their relatives' placement by their background characteristics as well as objective and subjective primary stressors. Their well-being may be affected simultaneously by nursing home stressors, e.g., communication with nursing assistants, activity participation, and interactions with relatives, staff, and other families, as well as CGs' and CRs' adjustments to a nursing home (Whitlatch et al., 2001).

Concepts such as person-centered care and advocacy, specifically for people with dementing illness, have been prominent since the 1990s, but expectations for QoL for people with physical and mental health issues have been refined in recent years as the concept and its measurement 
continue to be developed. This study reviews and synthesizes the research literature on the QoL of both CGs and their CRs with dementia after admission to long-term care facilities. Our conclusions about influences on QoL as well as QoL itself may provide guidance for identifying best practices, program reforms, and health and social policies.

\section{Review methods}

\section{Search procedure}

Ageline, MEDLINE, EBSCO, and PsycINFO electronic databases were used to search for studies using the terms placement, nursing home, SNFs, institutionalization, long-term care, small-scale housing, or group living in combination with the terms dementia or Alzheimer's disease and quality of life. The titles and abstracts of each identified study were scanned for relevance, and the reference lists from relevant articles were searched. The search excluded books, chapters, reports, and commentaries. The initial literature search yielded 78 articles.

\section{Selection criteria}

The current literature aims to examine the QoL of both family CGs and the dementia patients following placement. In order to achieve our goal, the following selection criteria were applied. First, the study must have been published in an English-language peer-reviewed journal. Second, in order to capture the most recent and relevant studies, we restricted our search to studies published within 15 years (i.e. 2002 or later). Third, CRs in the selected studies must have been diagnosed with $A D$ or a related dementia and must have been living in an institutional environment. Fourth, the study design must have been longitudinal (in order to understand the effects of placement) or a mixed-method study (in order to understand the perceived changes in QoL). Fifth, the study must have published at least one outcome measure based on a broad concept of QoL (Logsdon et al., 2002) for the CGs or CRs following placement, such as burden, depression, or some other measure of psychological well-being.

In total, 58 studies from the initial search were excluded for the following reasons: the reliability/ validity of measurement was questionable $(n=6)$; the studies were reviews $(n=8)$; the study's focus was on the effects of nursing home interventions $(n=7)$; the researchers studied the physical, psychological, or environmental determinants of the CR's QoL in general without assessing QoL after admission $(n=11)$; the studies combined samples, including day care, day hospital facilities, and clinics $(n=12)$; the study focus was on the quality of the care facilities $(n=11)$; the researchers focused on burden and compared nursing homes and other settings to extrapolate QoL data for the CGs with CRs in nursing homes $(n=3)$; the study outcome was CG satisfaction with nursing home placement $(n=1)$; or the studies looked at the effects of family involvement on caregiving $(n=3)$. Finally, after applying these inclusion and exclusion criteria, 12 studies remained, which focused on QoL of CGs $(n=5)$, CRs with dementia-related disorders in an institutional environment $(n=6)$, and both CGs and CRs $(n=1)$.

\section{Analyses}

Cohen and Weisman (1991) developed personal environment concepts for people with dementia living in group residential environments. Their conceptual framework for organizing a personal environment system, which we adopted in this review, included such components as patients' characteristics and the physical settings (Cohen and Weisman, 1991). Additionally, we evaluated the methodology of each study by the following criterion: use of outcome measures with demonstrated reliability and validity (Gingerich and Eisengart, 2000; Papp et al., 2009). Finally, effects/consequences of the placement on QoL of CG or CR were analyzed.

In the present review, each study was analyzed with respect to the following five domains (see Tables I and II):

- respondents' characteristics (e.g. age, race, CRs' scores on the Mini-Mental State Exam (MMSE));

- study design (e.g. sample size, mixed-method or quantitative); 


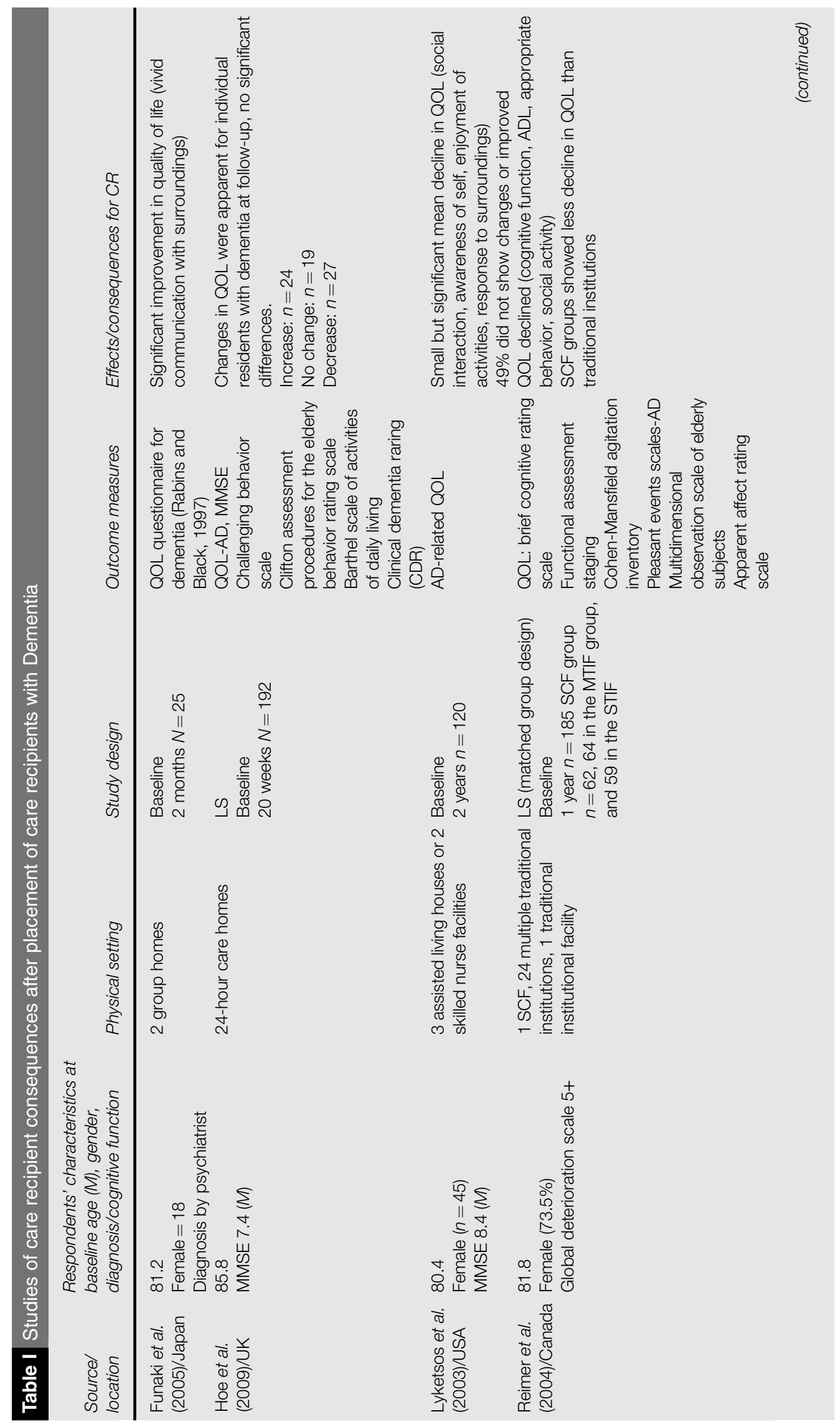




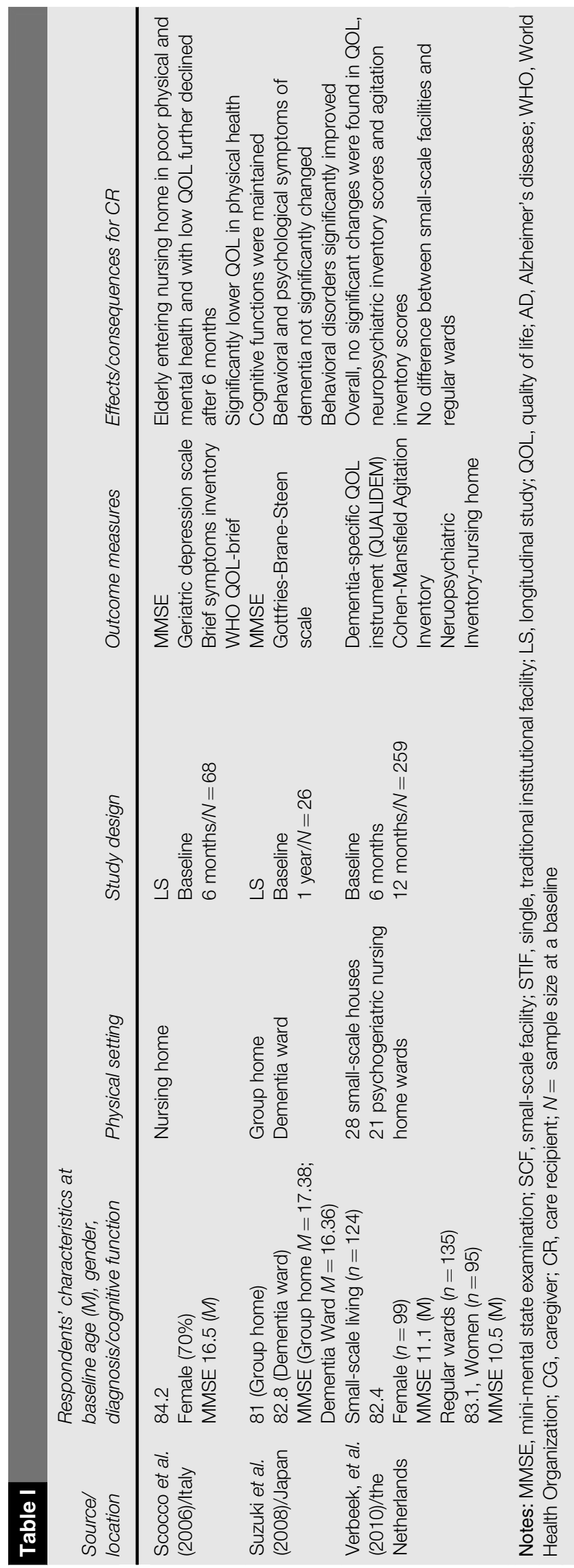




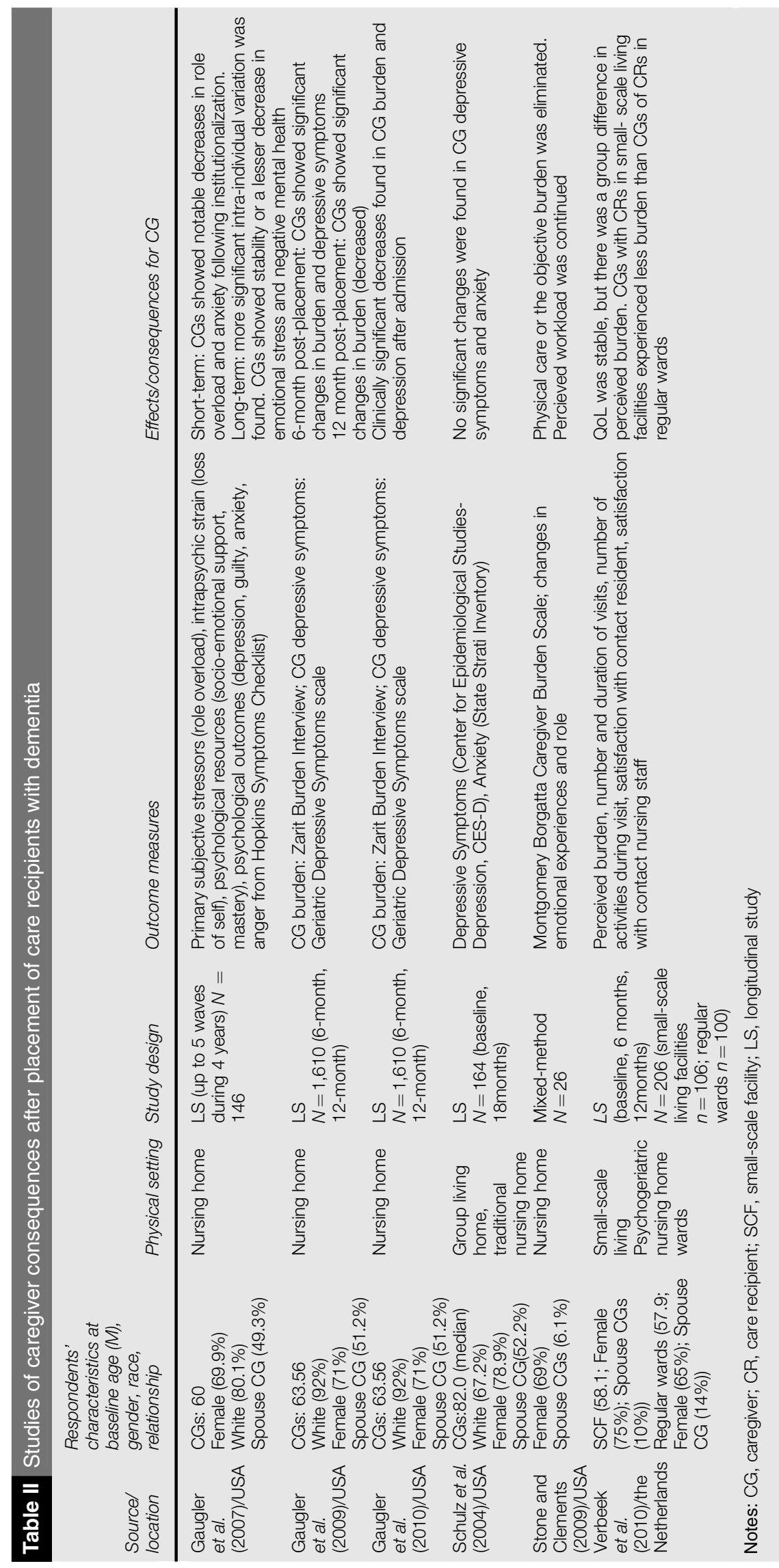


- physical setting (e.g. nursing home, group home);

- outcome measures (e.g. Zarit Burden Scale, brief cognitive rating scale); and

- effects/consequences (e.g. improvement in overall QoL).

\section{Findings}

\section{Studies of CRs' consequences after long-term care placement}

Characteristics. As seen in Table I, respondents were predominantly women with dementia. Mean age ranged from 81.2 to 85.8 years. Despite various ranges of MMSE (mean score from 7.4 to 16.5 at a baseline), MMSE was frequently used to select individuals with dementia as eligibility criteria, except for four studies: Funaki et al. (2005) used diagnosis of dementia by a psychiatrist; Lyketsos et al. (2003) used the Diagnostic and Statistical Manual of Mental Disorders; Reimer et al. (2004) used the global deterioration scale; and Suzuki et al. (2008) used MMSE to evaluate changes in scores after admission to long-term care facilities (e.g. group homes, 24-hour care homes, assisted living houses, group living, and nursing homes), but did not use it for eligibility criteria.

Study design. Sample sizes varied from 13 to 164 . Among seven studies with CR outcomes, two studies were conducted in Japan (Funaki et al., 2005; Suzuki et al., 2008), one in the UK (Hoe et al., 2009), one in the USA (Lyketsos et al., 2003), one in Canada (Reimer et al., 2004), one in Italy (Scocco et al., 2006), and one in the Netherlands (Verbeek et al., 2010). Most studies identified the informant (e.g. proxy, such as CGs, researchers, or nurse) with the exception of Lyketsos et al. (2003). Follow-up periods were from one month (Suzuki et al., 2008) to two years (Lyketsos et al., 2003).

Physical setting. Most studies recruited participants from more than one facility, except Scocco et al.'s (2006) study, which restricted its sample to a single nursing home with 383 beds. Various settings were included, such as group homes, 24-hour care homes, assisted living houses, nursing homes/traditional institutions, and dementia wards. Two studies (Reimer et al., 2004; Suzuki et al., 2008) compared the QoL of residents in different settings. Verbeek et al. (2010) investigated the experiences of family CGs in addition to those of residents. In this section, we focused on residents' experiences.

Outcome measure. The reviewed studies used standardized outcome measures. Only two studies (Funaki et al., 2005; Lyketsos et al., 2003) used a single measure to assess QOL; the other studies assessed additional aspects, such as memory function, depressive symptomatology, or physical function (see Table I).

Effects/consequences of placement. The effects of long-term care admission on residents' QoL varied across studies. Four studies showed a decreased QoL after admission. In particular, Lyketsos et al.'s (2003) study, at the two-year follow-up, showed a small but significant mean decline in QoL, specifically in social interaction, awareness of self, and enjoyment of activities. This study also found that residents who reported a lower baseline QoL were likely to show a greater decline in QoL at the two-year follow-up.

Reimer et al. (2004) found that QoL in cognitive function, activities of daily living, appropriate behavior, and social activity declined. In comparing the QoL of residents in special care facilities with that of residents in traditional facilities, this study found that residents in special care facilities showed less decline in activities of daily living, more sustained interest in the environment, and fewer negative effects than did residents in traditional institutions. Scocco et al. (2006) reported that at the six-month follow-up nursing home residents showed worsened cognitive function (as measured by MMSE), higher dependency levels in activities of daily living, and worsened QoL in the physical domain. Clinical symptoms such as somatization, obsessive/compulsive behavior, interpersonal sensitivity, depression, paranoid ideation, and geriatric depression were worse among women, with a 33 percent mortality rate.

Three studies showed that patients' QoL was stable or improved after admission. Funaki et al. (2005) investigated changes in QoL and factors related to the changes. Their findings indicated 
that group home residents enjoyed an improved QoL three months later. Admission in particular, a significant difference was observed in the QOL questionnaire subscale "vivid communication with surroundings." Suzuki et al.'s (2008) study found that cognitive function and behavioral and psychological symptoms of dementia among group home residents were stable at the one-year follow-up and that the residents reported significantly improved scores on activities of daily living and behavioral impairment. For the dementia care ward control group, the score for dressing unaided significantly decreased at the one-year follow-up (behavioral and psychological symptoms were not observed).

The evaluation study by Verbeek et al. (2010) found changes in QoL, neuropsychiatric symptoms and agitation differed for residents in small-scale facilities compared with control groups at the six- and 12-month follow-ups. The findings indicated that the residents in small-scale facilities showed decreased QoL as well as decreased neuropsychiatric symptoms and agitation, despite no statistically significant differences between residents in small-scale facilities at baseline and the 12-month follow-up. Furthermore, this study showed no significant group effect on QoL or neuropsychiatric symptoms and agitation.

Hoe et al. (2009) found mixed results from their assessment (at 20 weeks) of QoL and predictors of change in QoL in care home residents: although QoL showed no significant changes, individual scores reported by residents showed that QoL was stable for 19 residents and improved for 24 residents. However, individual scores reported by staff members showed that 27 residents had decreased QoL.

\section{Studies of caregivers' consequences after CRs' long-term care placement}

Characteristics. As seen in Table II, respondents were again predominantly women, primarily a spouse or an adult child. The mean age ranged from 57.9 to 63.56 years; one mixed-method study did not identify the mean age of CGs (Stone and Clements, 2009).

Study design. Sample size varied from 26 to 1610 at baseline. Four studies were conducted in the USA (Gaugler et al., 2007, 2009, 2010; Schulz et al., 2004; Stone and Clements, 2009), and one in the Netherlands (Verbeek et al., 2010).

Most studies were longitudinal, except for that of Stone and Clements (2009); follow-up periods varied from six months (Gaugler et al., 2009, 2010; Verbeek et al., 2010) to four years (Gaugler et al., 2007). Some studies assessed CGs' QoL, or burden, more than one time (Gaugler et al., 2007, 2009, 2010; Verbeek et al., 2010). However, although Gaugler et al. (2007) included pre-placement data as a baseline, the length of time between the baseline and two-wave post-placement was not indicated.

Physical setting. Most studies recruited the CGs of people with dementia at long-term care settings. Two groups of researchers included participants from only one type of facility, mainly nursing homes (Gaugler et al., 2007, 2009, 2010; Stone and Clements, 2009).

Outcome measures. As seen in Table II, six studies used multiple outcome measures to assess any changes in psychological distress, physical burden, or overall QoL. Most measures used in the six studies were well-developed and widely used to assess CGs' burden, although one mixed-method study also included an open-ended question "Describe how the caregiving sense of burden has changed for you since the placement of your loved one into a nursing home" (Stone and Clements, 2009, p. 204).

Effects/consequences of placement. The effects of long-term care admission on family CGs varied across studies. Gaugler et al. $(2009,2010)$, who investigated the effects on family CGs at six months post-placement (2009) and 12 months post-placement (2010), reported positive effects on family CGs' QOL after admission: CGs in both studies reported a significant decrease in burden and depressive symptoms following patients' nursing home admission compared with pre-placement. The first study (2009) explored the predictors of change in burden and depressive symptoms at six months after institutionalization, with six- and 12-month post-placement panels; this study found that higher levels of burden and depressive symptoms, the location (Florida), and 
more overnight hospital stays were significantly related to a worse sense of burden or depressive symptoms after patients' admission to a nursing home. The second study found that women CGs were more likely than men to report persistent burden following placement.

Three studies reported mixed findings. Gaugler et al. (2007) found that, for short-term effects (waves two and three), CGs showed notable decreases in role overload, anxiety, and anger following placement of their CRs. Over longer time periods (waves four and five), significantly more intra-individual variation was reported. CGs showed stability or a smaller decrease in emotional stress and negative mental health. Stone and Clements's (2009) mixed-method study CGs reported that the physical care or objective burden was eliminated, although perceived workload continued. The respondents were satisfied with their relatives' placement and the majority indicated their sense of burden shifted from concerns about providing physical care to providing more emotional and psychological support.

Finally, two studies reported no significant changes in CGs' QoL. Schulz et al.'s (2004) CGs indicated no changes in overall depressive symptoms and anxiety scores between pre- and post-placement. The researchers also noted that CGs who fell into one of three groups (spouses, CGs who visited at least daily, or CGs who were less satisfied with help from others) reported high levels of depressive symptoms after placement. Also, CGs who visited at least daily or who were less satisfied with help from others reported higher levels of post-placement anxiety. Verbeek et al. (2010) investigated the interaction between burden and setting in family CGs of residents in small-scale facilities and regular wards, and found that, although both groups remained stable over time in perceived burden, a significant group effect was found: family CGs of CRs in small-scale facilities reported less burden than family CGs of those in regular wards.

\section{Discussion}

The purpose of the current study is to provide a careful review of the effects of long-term care placement on the QoL for dementia CRs and their CGs. This review of 12 studies published between 2002 and 2013 reveals a discrepancy in the effects of institutionalization on the QoL of CRs and CGs. The results showed that among seven studies on change in CRs' QoL, three found a significant decline in CRs' QoL after placement (Lyketsos et al., 2003; Reimer et al., 2004; Scocco et al., 2006). Only one study showed that CRs' QoL was significantly improved (Funaki et al., 2005) and others showed that CR's QoL was stable (Hoe et al., 2009; Suzuki et al., 2008; Verbeek et al., 2010). Despite the variability, it is noteworthy that CRs in small-scale facilities such as a group home or a specialized care setting, which focuses on person centered care environment showed better outcomes. Surprisingly, studies of QoL changes in CGs showed much the same results: some family CGs benefited from placement (Gaugler et al., 2007, 2009, 2010; Stone and Clements, 2009), while others maintained their QoL (Schulz et al., 2004; Verbeek et al., 2010). However, after institutionalization, family CGs were more likely to report an improved QoL than were their CRs. Overall, despite continued caregiving involvement after CRs' institutionalization, these family CGs showed decreased objective burden. In particular, the types of facilities (small-scale living facilities) and CG gender (male) moderated the negative effects of CRs' institutionalization on CGs' QoL.

\section{Conceptual and clinical issues/implications}

First, attention should be paid to the dyadic experience of both CGs and CRs. None of the reviewed studies focused on any change to or patterns of the inter-relationships between CGs' and CRs' QoL, although one study (Verbeek et al., 2010) examined separately the change in CGs' and CRs' QoL. Despite a few exceptions (e.g. Whitlatch et al., 2006), the dyadic nature of caregiving has been relatively neglected in the literature. The change that occurs as dementia progresses may influence both inter-individual and intra-individual factors between the CGs and CRs, such as their relationship and the reciprocal interaction therein, which may in turn impact their QoL. Although the transition to an institution may yield stressors that operate differently for CRs and CGs (Aneshensel et al., 1995; Gaugler et al., 2010), because CGs and CRs share the same dyadic context, caregiving research must investigate the dynamics of the dyad interaction and its effects on both parties' QoL to better understand the post-admission caregiving situation. 
Future studies should examine the effects of the CG-CR relationship and CG-CR dyads' personal factors on changes in QoL after the CR's admission to long term care.

Second, with worsening cognitive function, concurrent physical declines and changes in behavior, most dementia patients often require long-term care placement. It is very important to understand the changes in various aspects of QoL and to maximize QoL for both CGs and CRs. Maximizing QoL during the period of institutional care is necessary for both CRs and CGs since, despite a decrease in CGs' objective burden (Gaugler et al., 2010), the need for post-admission caregiving to be provided by the CG may not have changed, but merely shifted in nature. We excluded studies investigating CR's QoL in general. Assessing the QoL of both CGs and CRs is important to provide a chance for both to express their thoughts about caregiving life before and after institutionalization. Measuring QoL can be useful to assess whether a transition or a service provider made a clinically significant difference (Logsdon et al., 2002).

Lastly, given the lack of longitudinal studies on changes in QoL in may help service providers implement appropriate interventions to maintain or enhance the QoL of both CGs and CRs (Logsdon et al., 2002), which may also impact the quality of care in long-term care facilities. The CG's individual characteristics also should be considered; some CG have become so deeply committed to the "caregiving career" that relinquishing the role is another loss which acts after placement to keep that CG involved and worried about their loved one. Understanding an individual's adaptation to placement could help service providers to provide more individual specific and high quality care. Also, because dementia is a progressive illness, CRs can be expected to have decreasing cognitive abilities coupled with potential worsening behavior, but an individual's progression will be idiosyncratic and unique. Consequently, depending on the measures studies used to define QoL, results may differ. Hopefully adequate medical and social management on a daily basis should help keep the patient in as good shape as possible and that is what some of the positive results for QoL suggest.

\section{Methodological/research issues and implications}

First, the differences in the effects of institutionalization on the QoL of CGs and CRs found by this review suggest that stressors may operate differently for the two groups. Thus, while family CGs and CRs face similar emergent challenges - e.g., how often the CG should visit the facility, how to interact with staff and each other in a new setting, how much supplemental assistance the CG should provide, redefining relationship with family and friends (Aneshensel et al., 1995) - there may be differences as well. Future studies should investigate these differences as well as the variability in changes for CGs and CRs due to the types of facility, quality of staff, individual characteristics, dyadic factors between CG and CR and other stressors.

Second, despite the increased interest in home-like facilities for dementia long-term care, many aspects regarding QoL remain unclear due to a lack of studies on the QoL of both CG and CR. Perhaps one reason that few studies have examined QoL in residential care for people with dementia is that, as O'Malley and Croucher (2005) argue, studies on aging have focused little on housing issues in general. Despite the importance of housing in dementia care, the two have been treated as separate areas for research, policy, and services. Also, long-term care settings such as RC/ALs have been studied only recently (Zimmerman et al., 2005). Future studies need to examine housing-related moderators and mediators of improvement in QoL after CR placement for the CGs as well as CRs.

Third, the differences in outcome measures, respondent characteristics, and the care settings found in this review make it very difficult to compare the outcomes reported by the reviewed studies. The outcomes reported are not comparable for a variety of reasons, including different QoL measurement scales, different CG and CR characteristics, and different care settings. Future studies should examine and report on the similarities and differences in the characteristics of the CG and the CR. Comparisons between different types of CGs and CRs, or different types of long-term care facilities, should be performed using a consistent and reliable set of standard outcome measures of the aspects of QoL, including behavioral competence, psychological status, physical functioning, and interpersonal environment (Logsdon et al., 2002).

Fourth, the immediate evaluation of the changes in CG and CR QoL, as well as the long-term trajectories, should be considered. One study (Funaki et al., 2005) conducted an immediate 
evaluation of the CR's QoL, but the majority of the other reviewed studies conducted assessments only between six months and two years after institutionalization of the CR. Without both the immediate and longer-term assessments, it is difficult to evaluate correctly the effects of placement, because recent events or changes in the lives of both CG and CR may the impact of placement being over- or under-estimated (Moon and Adams, 2013). In three studies (Gaugler et al., 2007, 2009, 2010), the assessment period was less than two years, which may affect confidence in the long-term effects of placement on CG and CR (Moon and Adams, 2013). Lastly, assessments of changes in QoL should be conducted over longer follow-up time periods, since patients with dementia stay in long-term care facilities significantly longer from entry until discharge or death than patients without dementia (Sabbagh et al., 2003). In particular, the average length of stay for nursing home residents with dementia is increasing, with more than one-third residing in nursing homes for three years or longer (Wilkins et al., 2005).

Finally, recruiting more minority participants is essential for future research. None of these studies focused on the experience of racial/ethnic minorities following placement, and few studies included non-whites. For example, Gaugler et al. recruited only 19.9 percent of their respondents from non-white ethnicities for their CG panel study (2007) and fewer still (8 percent) for their six-month (2009) and 12-month (2010) study of CRs. Schulz et al. (2004) selected only 31.8 percent of their respondents from non-white groups for their CG study. The older population in the USA is projected to become more racially and ethnically diverse over the next 50 years (US Census Bureau, 2008): the non-Hispanic white population, which constituted 80 percent of the population over 65 in 2010, is expected to decrease more than 20 percent from 2010 in 2050, while the proportions of the other ethnic groups are expected to grow on average 8 percent (e.g. African American from 8.3 to 11.2 percent, Asian from 3.3 to 8.5 percent, Hispanic from 7 to 20 percent, and Native American from 1 to 2 percent) in the same time period (US Census Bureau, 2008). It will also be beneficial to develop appropriate interventions based on the different cultural needs of each group. Along with a significant decrease in the ratio of family CG to older people, caring for older people (especially those with dementia, who may face institutionalization at some point) is a major public health problem. Coupled with the rapid increases in demographic diversity, this underlines a pressing need to understand the experience of placement in diverse ethnic groups.

\section{Conclusion}

Although we found differences in reported results for the QoL changes after the care receiver had a placement, this review shows that most care receivers with dementia experienced some level of change in their own QoL. On the other hand, most caregivers in the reviewed studies reported a decreased burden and lowered levels of emotional stress. Based on the studies reviewed, we recommend that both care receivers and caregivers would benefit from a person-centered and individualized needs-based residential care environment grounded in well-established research evidence. We also recommend that future studies should focus on the importance of understanding the individual's adaptation to placement and dyadic changes in QoL (including mediators/moderators). However, to implement these recommendations, both longitudinal and dyadic studies that include multi-ethnic populations are needed to better understand changes in QoL for both caregivers and care receivers following the long-term placement in care of the care receiver. Future findings from such studies will provide better evidence to address the needs of older people in residential care settings and their caregivers.

\section{References}

Alzheimer's Association (2014), "Alzheimer's disease facts and figures", Alzheimer's \& Dementia, Vol. 10 No. 2, pp. e47-e92, available at: www.alz.org/national/documents/report_alzfactsfigures2014.pdf (accessed December 2015).

Aneshensel, C.S., Pearlin, L.I., Mullan, J.T., Zarit, S.H. and Whitlatch, C.J. (1995), Profiles in Caregiving: The Unexpected Career, Academic Press, San Diego, CA.

Boekhorst, B.T., Pot, A.M., Depla, M., Smit, D., de Lange, J. and Eefsting, J. (2008), "Group living homes for older people with dementia: the effects on psychological distress of informal caregivers", Aging \& Mental Health, Vol. 12 No. 6, pp. 761-8. 
Brod, M., Stewart, A.L., Sands, L. and Walton, P. (1999), "Conceptualization and measurement of quality of life in dementia: the dementia quality of life instrument (DQoL)", The Gerontologist, Vol. 39 No. 1, pp. 25-36.

Cohen, U. and Weisman, G. (1991), Holding on to Home, Johns Hopkins University Press, Baltimore, MD.

Dobriansky, P.J., Suzman, R.M. and Hodes, R.J. (2007), "Why population aging matters: aglobal perspective", National Institute on Aging, National Institutes of Health, US Department of Health and Human Services, US Department of State.

Ferrans, C.E., Zerwic, J.J., Wilbur, J.E. and Larson, J.L. (2005), "Conceptual model of health-related quality of life”, Journal of Nursing Scholarship, Vol. 37 No. 4, pp. 336-42.

Funaki, Y., Kaneko, F. and Okamura, H. (2005), "Study on factors associated with changes in quality of life of demented elderly persons in group homes", Scandinavian Journal of Occupational Therapy, Vol. 12 No. 1, pp. 4-9.

Gaugler, J.E., Mittelman, M.S., Hepburn, K. and Newcomer, R. (2009), "Predictors of change in caregiver burden and depressive symptoms following nursing home admission", Psychology and Aging, Vol. 24 No. 2, pp. 385-96, doi: 10.1037/a0016052.

Gaugler, J.E., Mittelman, M.S., Hepburn, K. and Newcomer, R. (2010), "Clinically significant changes in burden and depression among dementia caregivers following nursing home admission", BMC Medicine, Vol. 8 No. 85, pp. 1-12, doi: 10.1186/1741-7015-8-85.

Gaugler, J.E., Pot, A.M. and Zarit, S.H. (2007), "Long-term adaptation to institutionalization in dementia caregivers", Gerontologist, Vol. 47 No. 6, pp. 730-40, doi: 10.1093/geront/47.6.730.

Gingerich, W. and Eisengart, S. (2000), "Solution-focused brief therapy: a review of the outcome research", Family Process, Vol. 39 No. 4, pp. 477-98.

Hoe, J., Hancock, G., Livingston, G., Woods, B., Challis, D. and Orrell, M. (2009), "Changes in the quality of life of people with dementia living in care homes", Alzheimer Disease \& Associated Disorders, Vol. 23 No. 3, pp. 285-90, doi: 10.1097/WAD.0b013e318194fc1e.

Kitrungrote, L. and Cohen, M.Z. (2006, May), "Quality of life of family caregivers of patients with cancer: a literature review”, Oncology Nursing Forum, Vol. 33 No. 3, pp. 626-32.

Lawton, M.P. (1994), "Quality of life in Alzheimer disease”, Alzheimer Disease \& Associated Disorders, Vol. 8 , pp. 138-50.

Logsdon, R.G., Gibbons, L.E., McCurry, S.M. and Teri, L. (2002), "Assessing quality of life in older adults with cognitive impairment”, Psychosomatic Medicine, Vol. 64 No. 3, pp. 510-19.

Lyketsos, C.G., Gonzales-Salvador, T., Chin, J.J., Baker, A., Black, B. and Rabins, P. (2003), "A follow-up study of change in quality of life among persons with dementia residing in a long-term care facility", International Journal of Geriatric Psychiatry, Vol. 18 No. 4, pp. 275-81.

O'Malley, L. and Croucher, K. (2005), "Housing and dementia care - a scoping review of the literature", Health \& Social Care in the Community, Vol. 13 No. 6, pp. 570-7.

Macdonald, A.J.D., Carpenter, G.I., Box, O., Roberts, A. and Sahu, S. (2002), "Dementia and use of psychotropic medication in non-'elderly mentally infirm' nursing homes in South East England", Age and Aging, Vol. 31 No. 1, pp. 58-64.

Moon, H. and Adams, K.B. (2013), "The effectiveness of dyadic interventions for people with dementia and their caregivers”, Dementia, Vol. 12 No. 6, pp. 821-39.

Papp, K.V., Walsh, S.J. and Snyder, P.J. (2009), "Immediate and delayed effects of cognitive interventions in healthy elderly: a review of current literature and future directions", Alzheimer's \& Dementia: The Journal of the Alzheimer's Association, Vol. 5 No. 1, pp. 50-60.

Prince, M., Prina, M. and Guerchet, M. (2013), "Journey of caring: an analysis of long-term care for dementia”, doctoral dissertation, Alzheimer's Disease International, London.

Rabins, P.V. and Black, B.S. (2007), "Measuring quality of life in dementia: purposes, goals, challenges and progress", International Psychogeriatrics, Vol. 19 No. 3, pp. 401-7.

Ready, R.E., Ott, B.R. and Grace, J. (2004), "Patient versus informant perspectives of quality of Ife in mild cognitive impairment and Alzheimer's disease", International Journal of Geriatric Psychiatry, Vol. 19 No. 3, pp. 256-65. 
Redfoot, D., Feinberg, L. and Houser, A. (2013), The Aging of the Baby Boom and the Growing Care Gap: A Look at Future Declines in the Availability of Family Caregivers, Insight on the issues, 85, AARP Public Policy Institute, Washington, DC, pp. 1-12.

Reimer, M.A., Slaughter, S., Donaldson, C., Currie, G. and Eliasziw, M. (2004), "Special care facility compared with traditional environments for dementia care: a longitudinal study of quality of life", Journal of the American Geriatrics Society, Vol. 52 No. 7, pp. 1085-92, doi: 10.1111/j.1532-5415.2004.52304.

Sabbagh, M.N., Silverberg, N., Majeed, B., Samant, S., Sparks, D.L., Seward, J. and Connor, D.J. (2003), "Length of stay in skilled nursing facilities is longer for patients with dementia", Journal of Alzheimer's Disease, Vol. 5 No. 1, pp. 57-63.

Schulz, R., Belle, S.H., Czaja, S.J., McGinnis, K.A., Stevens, A. and Zhang, S. (2004), "Long-term care placement of dementia patients and caregiver health and well-being", Journal of the American Medical Association, Vol. 292 No. 8, pp. 961-7, doi: 10.1001/jama.292.8.961.

Scocco, P., Rapattoni, M. and Fantoni, G. (2006), "Nursing home institutionalization: a source of eustress or distress for the elderly?", International Journal of Geriatric Psychiatry, Vol. 21 No. 3, pp. 281-7, doi: 10.1002/ gps.1453.

Sloane, P.D., Zimmerman, S., Williams, C.S., Reed, P.S., Gill, K.S. and Preisser, J.S. (2005), "Evaluating the quality of life of long-term care residents with dementia", The Gerontologist, Vol. 45 No. S1, pp. 37-49.

Stone, L.J. and Clements, J.A. (2009), "The effects of nursing home placement on the perceived levels of caregiver burden”, Journal of Gerontological Social Work, Vol. 52 No. 3, pp. 193-214, doi: 10.1080/ 01634370802609163.

Suzuki, M., Kanamori, M., Yasuda, M. and Oshiro, H. (2008), "One-year follow-up study of elderly group-home residents with dementia", American Journal of Alzheimer's Disease and Other Dementias, Vol. 23 No. 4, pp. 334-43, doi: 10.1177/153331750831915.

United Nations (2013), "World population ageing 2013", ST/ESASER.A/348, Department of Economic and Social Affairs, Population Division.

Verbeek, H., Zwakhalen, S.M., van Rossum, E., Ambergen, T., Kempen, G.I. and Hamers, J.P. (2010), "Dementia care redesigned: effects of small-scale living facilities on residents, their family caregivers, and staff", Journal of the American Medical Directors Association, Vol. 11 No. 9, pp. 662-70, doi: 10.1016 j.jamda.2010.08.001.

Whitlatch, C.J., Judge, K., Zarit, S.H. and Femia, E. (2006), "Dyadic intervention for family caregivers and care receivers in early-stage dementia", The Gerontologist, Vol. 46 No. 5, pp. 688-94.

Whitlatch, C.J., Schur, D., Noelker, L.S., Ejaz, F.K. and Looman, W.J. (2001), "The stress process of family caregiving in institutional settings”, Gerontologist, Vol. 41 No. 4, pp. 462-73, doi: 10.1093/geront/41.4.462.

Whoqol Group (1995), "The world health organization quality of life assessment (WHOQOL): position paper from the world health organization”, Social Science \& Medicine, Vol. 41 No. 10, pp. 1403-9.

Wilkins, C.H., Moylan, K.C. and Carr, D.B. (2005), "Diagnosis and management of dementia in long-term care", Annals of Long-Term Care, Vol. 13 No. 11, pp. 17-24.

Zarit, S.H. and Whitlatch, C.J. (1992), "Institutional placement: phases of the transition”, Gerontologist, Vol. 32 No. 5, pp. 665-72, doi: 10.1093/geront/32.5.665.

Zimmerman, S., Sloane, P.D., Williams, C.S., Reed, P.S., Preisser, J.S., Eckert, J.K., Boustani, M. and Dobbs, D. (2005), "Dementia care and quality of life in assisted living and nursing homes", Gerontologist, Vol. 45 No. S1, pp. 133-46, doi: 10.1093/geront/45.suppl_1.133.

\section{Further reading}

Bramble, M., Moyle, W. and McAllister, M. (2009), "Seeking connection: family care experiences following long-term dementia care placement", Journal of Clinical Nursing, Vol. 18 No. 22, pp. 3118-25, doi: 10.1111/ j.1365-2702.2009.02878.x.

Burgener, S. and Twigg, P. (2002), "Relationships among caregiver factors and quality of life in care recipients with irreversible dementia", Alzheimer Disease \& Associated Disorders, Vol. 16 No. 2, pp. 88-102. 
Sauvaget, C., Tsuji, I., Haan, M.N. and Hisamichi, S. (1999), "Trends in dementia-free life expectancy among elderly members of a large health maintenance organization”, International Journal of Epidemiology, Vol. 28 No. 6, pp. 1110-18.

US Census Bureau (2007), "State and county quickfacts", Allegany County, NY, available at: http://quickfacts. census.gov (accessed January 25, 2007).

van Zadelhoff, E., Verbeek, H., Widdershoven, G., van Rossum, E. and Abma, T. (2011), "Good care in group home living for people with dementia: experiences of residents, family and nursing staff", Journal of Clinical Nursing, Vol. 20 Nos 17-18, pp. 2490-500, doi: 10.1111/j.1365-2702.2011.03759.x.

Welsh, H.G., Walsh, J.S. and Larson, E.B. (1992), "The cost of institutional care in Alzheimer's disease: nursing home and hospital use in a prospective cohort", Journal of the American Geriatrics Society, Vol. 40 No. 3, pp. 221-4.

\section{Corresponding author}

Heehyul Moon can be contacted at: heehyulm@gmail.com

For instructions on how to order reprints of this article, please visit our website: 